\title{
Silver Nanoparticles: Synthesis and Applications
}

\author{
Mohd Yusuf
}

\section{Contents}

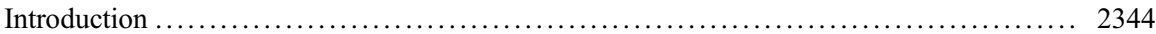

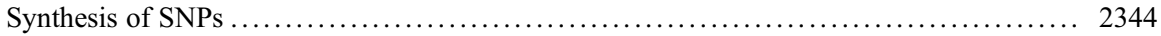

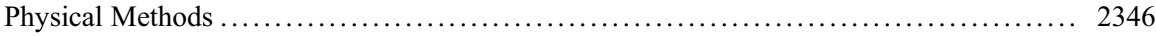

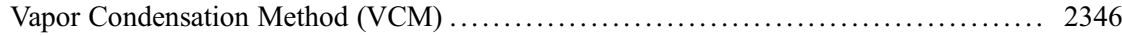

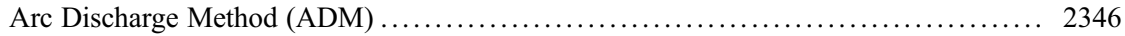

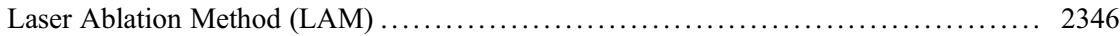

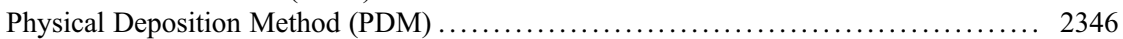

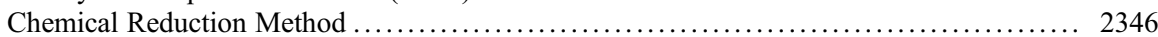

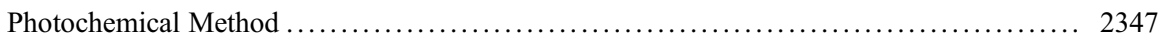

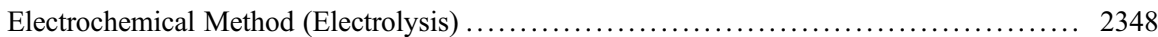

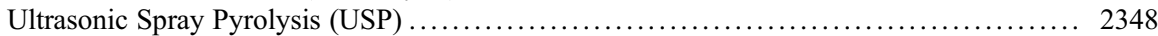

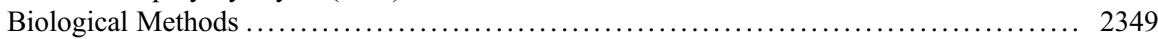

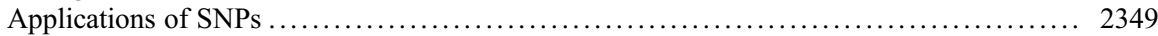

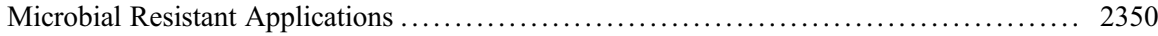

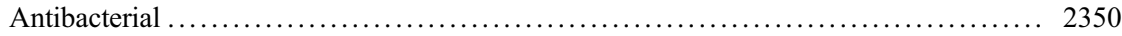

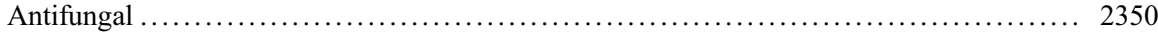

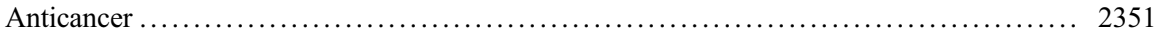

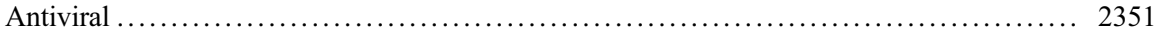

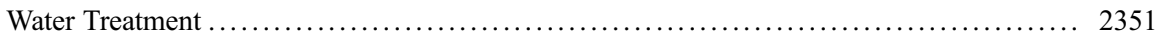

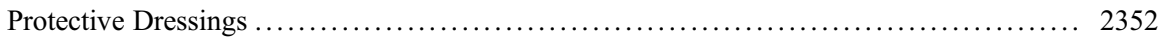

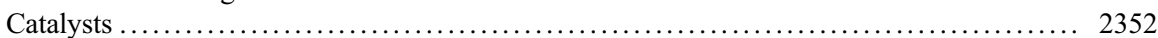

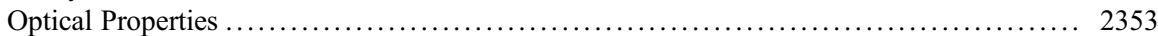

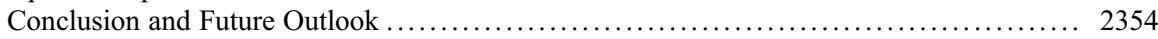

References ............................................................ 2354

M. Yusuf $(\varangle)$
Department of Chemistry, YMD College, Maharshi Dayanand University, Nuh, Haryana, India e-mail: yusuf1020@gmail.com 


\begin{abstract}
Nanotechnology involves the production, manipulation, and use of materials ranging in size from less than a micron to that of individual atoms, is an emerging field of modern science, and utilizes nanoscaled systems. Nanoparticles (NPs) are being viewed as fundamental building blocks of nanotechnology. Although nanomaterials may be synthesized using chemical approaches, it is now possible to include the use of biological systems such as bacteria, fungi, and plants. Currently, silver nanoparticles have shown great importance due to its large surface ratio and unique properties with myriad activities applicable in a number of applied sectors including microbial resistant applications (antibacterial/antifungal/anticancer/antiviral), water treatment, protective dressings, catalysis, and optical devices. This chapter encompasses the fabrication methods and dispersed applications of silver nanoparticles.
\end{abstract}

\title{
Introduction
}

In term "Nanotechnology," the prefix nano is adopted from a Greek word nanos which mean small or dwarf that refers to one-billionth $\left(10^{9} \mathrm{~m}\right)$ in size. Nanotechnology deals with the manufacturing and applications of materials with nanoscale diameter. Nanoparticles (NPs) have shown distinctive properties, which can be rightfully manipulated for several preferred applications due to their high surface area to volume ratio and vast electronic, optical, magnetic, chemical, and physical properties. Significant interest has arisen in the research of NPs during the last decade. Nanoparticles due to their controlled size and composition got fundamental and technological attention because they offer solutions to technological and environmental challenges, owe to their wide applications in various fields, such as in pharmaceutical and biomedical, biomedicine, catalysis, water treatment, energy conservation with tunable electrical conductivity, thermal conductivity, tensile strength, superior rigidity, hardness and erosion resistance, which are currently used for manufacturing of satellite components, aircraft spares, industry parts, and electronic microchips $[1,2]$. However, in most studies they are suggested to be nontoxic, but due to their small size and variable properties, they are suggested to be perilous to the environment to a significant extent [3]. The present chapter highlights the various synthetic schemes and diversified applications of silver nanoparticles (AgNPs).

\section{Synthesis of SNPs}

AgNPs have been shown to have an excellent microbial resistant ability. Therefore, they have become a part of our daily life, for example, being present in clothes, household and personal care products, mainly due to their antimicrobial properties. 
In general, the methods used for the generation of metallic AgNPs are divided into two main processes, namely top-down or bottom-up [4]. To understand these processes in a lucid manner, the Brick Concept may be taken. Breaking a wall down into its components, the bricks refer to the top-down approach, whereas fabrication of the brick from initial ingredients like clay-bearing soil, sand, lime, and water would represent bottom-up approach (Figs. 1and 2). Consequently, in a systematic way of nanoscience, the top-down approach involves the use of bulky materials and reduces them into nanoparticles via physical/chemical/mechanical processes. On the other hand, the bottom-up approach requires begins from molecules or atoms to obtain nanoparticles [3]. Some most frequently used methods for the synthesis of AgNPs are discussed herein (Fig. 2).

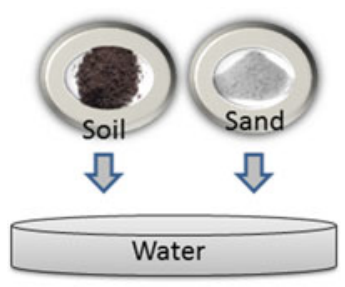

Bottom-up
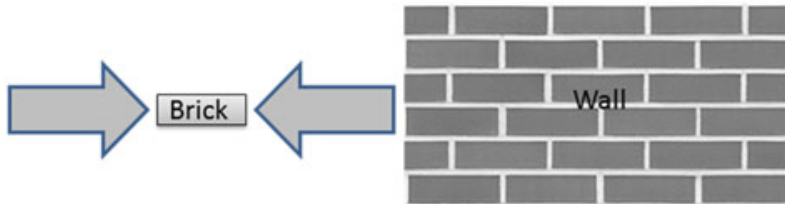

Top-Down

Fig. 1 Illustrative representation of NPs synthesis through bottom-up and top-down processes using Brick Concept

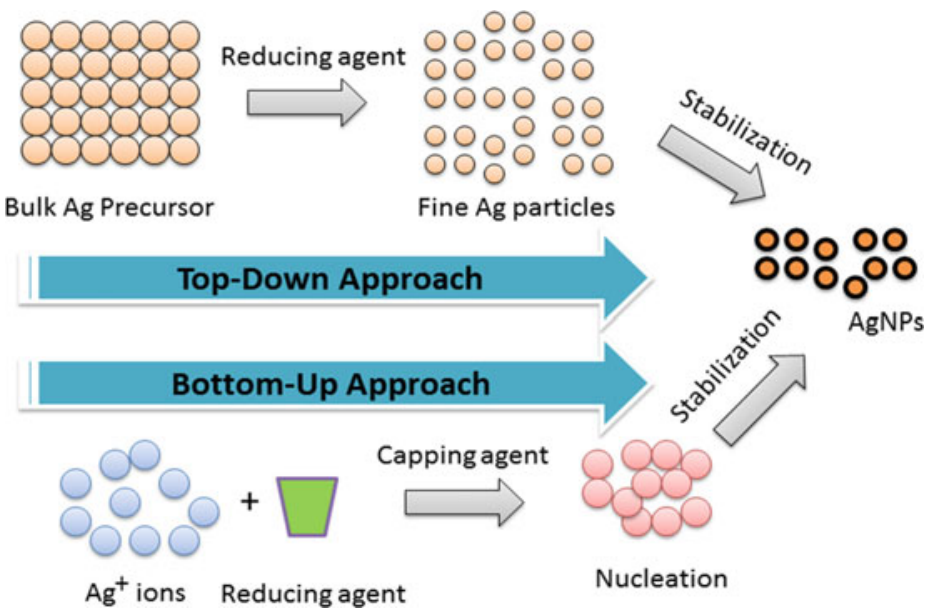

Fig. 2 Schematic representation of AgNPs synthesis through bottom-up and top-down processes 


\section{Physical Methods}

\section{Vapor Condensation Method (VCM)}

Vapor condensation process involves two steps, evaporation and condensation, which are carried out using a tube furnace at atmospheric pressure. The source material within a boat centered at the furnace is vaporized into a carrier gas. Nanoparticles of various materials, such as $\mathrm{Ag}, \mathrm{Au}, \mathrm{PbS}$, can be prepared by this method. In order to synthesize AgNPs, the vaporization method has been frequently used, in which the target materials are vaporized by the heat source and then rapidly condensed resulting in the synthesized nanoparticles.

\section{Arc Discharge Method (ADM)}

Arc discharge method for the synthesis of nanoparticles is an efficient method in which a direct-current arc voltage is applied across two graphite electrodes immersed in an inert gas such as $\mathrm{He}, \mathrm{Ar}$, or Ne. It is a novel technique for preparing a nanosilver water suspension without surfactants, and stabilizers was studied using the arcdischarge method. During the arc-discharge, the surface layer of the Ag wires evaporates and condenses in the water layer. Thereafter, the transparent solution converts to a characteristic pale yellow color which turns golden-brown colloidal suspension of silver nanoparticles.

\section{Laser Ablation Method (LAM)}

Silver nanoparticles can be synthesized with laser ablation onto metallic bulk silver salts in solution phase. Laser ablation method has the advantage compared to other conventional method for preparing metal colloids is the absence of chemical reagents in solutions. Therefore, pure colloidal AgNPs are produced by this method.

\section{Physical Deposition Method (PDM)}

More recent process for the fabrication of AgNPs is physical deposition method. This unique technique is capable of the physical synthesis of AgNPs with uniform geometry and particle size less than $5 \mathrm{~nm}$ along with improved dispersion.

\section{Chemical Reduction Method}

To synthesize silver nanoparticles by chemical reduction is the most frequently applied method for the preparation of stable, colloidal dispersions in water or other suitable organic solvents. In general, the chemical reduction process of AgNPs in the 
solution includes the following three main components: (i) metal precursors, (ii) reducing agents, and (iii) stabilizing/capping agents. The overall phenomenon involves coprecipitation in which the concurrence of different substeps such as reduction, nucleation, growth, coarsening, and agglomeration observed. In this strategy, the size is controlled by arrested precipitation technique. The basic trick has been to synthesize and study the nanomaterial in situ, that is, in the same liquid/ medium avoiding the physical changes and aggregation of tiny crystallites or particles. At this stage, a capping agent is introduced to the parent solution before precipitation reaction to maintain separation between the particles formed. As prepared, nanoparticles are separated by centrifugation, washed and vacuum dried. The dried material was further subjected to UV curing for the possible polymerization of surfactant capping film on the surface of nanocluster for imparting true quantum confinement [5]. There are several typical reducing agents include $\mathrm{NaBH}_{4}, \mathrm{~N}_{2} \mathrm{H}_{4}$, polyols, tri-sodium citrate (TSC), sodium citrate, and N,N-dimethylformamide (DMF). In order to prevent aggregation between AgNPs, sodium dodecyl sulfate (SDS) and some polymeric systems like polyvinyl pyrrolidone (PVP), polymethacrylic acid, and polymethylmethacrylate have been reported to be the effective protective agents to stabilize as capping agents for stabilization. Some of the chemical reducing reactions can be carried out at ambient temperature, while most of them need elevated temperatures for increasing reaction rate.

However, nanoparticle morphologies strongly influenced by the temperature variant were adopted during the synthesis. AgNPs show much variation in shape ranging from spherical to trigonal/hexagonal. Ascorbic acid as a reducing agent favors the formation of flower-like silver nano architecture at room temperature with average particle size of $20 \mathrm{~nm}$. Moreover, AgNPs have been synthesized by the polyol process with the assistance of supercritical carbon dioxide from nitrate salt of silver as the base material, polyvinyl pyrrolidone (PVP) as the stabilizer for the silver clusters, and ethylene glycol as the reducing agent and solvent. PVP not only protected the nano-sized silver particles from aggregation, but it also promoted nucleation phenomenon [6,7]. Moreover, for the preparation of monodispersed and uniform sized ANPs, the simultaneous formation of nuclei for subsequent growth is required. The initial nucleation and the subsequent growth of nuclei can be controlled by adjusting the reaction parameters such as temperature, $\mathrm{pH}$, precursor types, solvents, reduction agents, and stabilizing/capping agents.

\section{Photochemical Method}

AgNPs also can be successfully synthesized by using a variety of photo-irradiation processes. Albeit less practiced photo-assisted synthesis of silver used for the preparation of AgNPs allows the preparation of stable AgNPs by irradiation of a reaction mixture with a light source such as laser or lamp in the presence of photoreducing agents without the need to introduce stabilizers or surfactants [8-10]. For example, laser irradiation of an aqueous solution of Ag salt and surfactant can fabricate stable AgNPs with well-dispersed shape and size distribution. 
AgNPs with narrow size distribution were synthesized in ethylene glycol-water solvent systems without the use of a stabilizer. They used pulse radiolysis method to produce nanoparticles by silver perchlorate salt. Reduction of $\mathrm{Ag}^{+}$to $\mathrm{Ag}^{0}$ was achieved successfully using UV light instead of chemical materials in a natural rubber matrix via photo reduction of the film cast from natural rubber latex (NRL) containing silver salt, with an average size ranged $10 \mathrm{~nm}$. Synthesis procedures using microwave irradiation have also been employed, and this method is known to have a faster heating rate than conventional heating through conduction and convection. Their size and the time needed for their preparation are directly proportional to the irradiation power of the light source. For example, with a low-power lamp (4 W), irradiation for $9 \mathrm{~h}$ is needed to produce $19 \mathrm{~nm}$ diameter AgNPs, while with a stronger source $(150 \mathrm{~W})$, the reaction takes only $45 \mathrm{~min}$. However, in the latter case, the AgNPs are polydisperse and smaller than when they are prepared using a lowerenergy lamp [11].

\section{Electrochemical Method (Electrolysis)}

The electrolysis process has long been employed for the preparation of various NPs from their respective metal ions. In this method, a rotating disk electrode is used as the cathode, and a plate is used as the anode, which resulted in the formation of electro-deposited $\mathrm{Ag}^{0}$ nanoparticles. Reetz et al. described an electrochemical procedure to obtain particles in which a metal sheet is anodically dissolved and the intermediate metal salt formed is reduced at the cathode, giving rise to metallic particles stabilized by tetraalkylammonium salts. This method has several advantages, like the high purity of the particles and the possibility of a precise particle-size control achieved by adjusting the current density [12]. Rodriguez-Sanchez generated feasible nanoparticles ranging from $2 \mathrm{~nm}$ to $7 \mathrm{~nm}$ having silver plasmon band that appears at about $370 \mathrm{~nm}$ based on the dissolution of a metallic anode in an aprotic solvent, which has been used to obtain silver [13]. Recently, Nasretdinova et al. reported an efficient synthesis of AgNPs stabilized by cetyltrimethylammonium cations (Ag@ $\mathrm{CTA}^{+}$) carried out in aqueous medium by methylviologen-mediated electroreduction of silver chloride nanospheres stabilized by surface-active $\mathrm{CTA}^{+}$ cations (AgCl@CTA ${ }^{+}$, diameter $330 \mathrm{~nm}$ ), on a glassy carbon electrode at potentials of the $\mathrm{MV}^{2+/} \mathrm{MV}^{\bullet+}$ redox couple [14].

\section{Ultrasonic Spray Pyrolysis (USP)}

Ultrasonic spray pyrolysis is a convenient, innovative, and powerful tool for the synthesis of nanoparticles with controlled and uniform particle size [15]. This technology has a great potential to be the future solution for the synthesis of silver nanopowder. In this process, a metal-containing solution is cold-atomized which forms an aerosol and enables an easy control of the powder morphology and the excellent availability of cheap precursors at acceptable costs. Chemical processes 
synthesizing metal nanoparticles usually involve toxic chemicals, which are harmful to our health and environment. PSP method has the advantage over chemical reduction methods and successfully produces pure metallic nanoparticles. Ultrasonic spray pyrolysis may be employed to generate an aerosol from a dilute aqueous metal salt solution, resulting in the production of particles with a narrow size distribution. Pingali et al. reported silver nanoparticles having an average size of less than $20 \mathrm{~nm}$ in diameter by pyrolysis of an ultrasonically atomized spray of highly dilute aqueous silver nitrate solution at temperatures above $650{ }^{\circ} \mathrm{C}$ and below the melting point of silver [16]. Additionally, the independent variables of solution concentration and atomizer ultrasound power have shown to have a significant impact on the size distribution of nanoparticles produced.

\section{Biological Methods}

The potential of plants as a source of drugs and herbal remedied as well is largely explored, since antiquity. Historically, pharmacological screening of compounds of natural origin has been the source of innumerable therapeutic agents, and it is variously estimated that about 8,000 natural antibiotics have been isolated, characterized, synthesized, and noticed to possess potent biological activities, around the globe. Researchers pay attention toward the use of plants with potential in phytomining and phytoremediation of metals in order to phytosynthesizing metallic nanoparticles and thus often opening of a new innovation occurred in fast and nontoxic methods for production of nanoparticles [2]. In general, nanoparticles are prepared by a variety of chemical and physical processes which are quite expensive and potentially hazardous to the environment that involves the use of toxic and unsafe chemicals that are responsible for various environmental risks. So, new safe alternatives were searched for the fabrication of nanoparticles. Consequently, the development of biologically inspired experimental processes for the syntheses of nanoparticles is evolving into an important branch of nanotechnology. Living organisms that are referred to potent nano factories for fabrication of silver nanoparticles such as bacteria, fungi, plants, and biomass have huge potential for the production of metal nanoparticles $[17,18]$. Today, the development of feasible and reliable green processes for the synthesis of silver nanoparticles is an important aspect of current nanotechnology research.

\section{Applications of SNPs}

As we know, production of nanoparticles can be achieved through different methods. Silver nanoparticles are one of the most attractive nanomaterials for commercialization applications. They have been used extensively as electronic products in the industry and antibacterial agents in the health industry. Explored applications of AgNPs are discussed as under: 


\section{Microbial Resistant Applications}

\section{Antibacterial}

Antibacterial effect against anaerobic and aerobic bacteria of AgNPs is widely studied by different scientist and research communities around the world. AgNPs show highly antibacterial effect due to their formation of free radicals on its surface to penetrate through the membrane of the cell to disturb the intracellular processes [19]. Furthermore, Furno research group has demonstrated the potential of silver nanoparticles as an antimicrobial agent. They showed that silicon discs impregnated with silver nanoparticles efficiently prevented bacterial adhesion and growth. Their potent antibacterial activity was reported against various strains of bacteria including highly pathogenic bacteria species including gram positive and gram negative bacteria. It was observed that a small amount of AgNPs is deadly for the majority of viruses and bacteria [20]. Sondi and Salopeck-Sondi reported the antibacterial activities of AgNPs against E. coli (representative species for gram negative bacteria) on Luria-Bertani agar plates [21]. In another study, Morones et al. reported experiments on the size-related properties of the AgNPs on different species of gram negative bacterial strains. The results obtained from their study suggested that size of AgNPs is an important factor in preventing the bacterial cells from their normal functions [22]. Recently, Sonker et al. investigated an eco-friendly, green, cheap, and convenient biological method for the synthesis of AgNPs using the cell extract of the cyanobacterium Nostoc sp. strain HKAR-2. As prepared AgNPs showed a dose-dependent cytotoxic activity against human breast cancer MCF-7 cells with $\mathrm{IC}_{50}$ of $27.5 \mu \mathrm{g} / \mathrm{ml}$ and therefore exhibited excellent antibacterial and antifungal activities [23].

\section{Antifungal}

Pathogenic fungi are those fungi that cause diseases in living organisms and humans and are considered to play a vital role in causing fungal infections, especially in hospitals. Although fungi are eukaryotic organisms, on the other hand, many pathogenic fungi are also microorganisms. The study of pathogenic fungi is referred to as Mycology. Along with the antibacterial activities of the AgNPs, numerous studies had been reported on the antifungal efficacy of AgNPs, which reveal that AgNPs could be used as an effective antifungal agent because AgNPs exhibit excellent antifungal characteristics against a number of fungal species. In a study, Kim et al. reported the antifungal activities of the AgNPs against different fungal strains such as Trichophyton mentagrophytes and Candida albicans fungi and observed that AgNPs exhibited good antifungal activity [24]. Later, the antifungal activity of the silver nanoparticles fabricated by the modified Tollens process was evaluated for common pathogenic Candida sp. by in terms of the determination of the minimum inhibitory concentration (MIC), minimum fungicidal concentration (MFC), and the time-dependency of yeasts growth inhibition reported by Panacek et 
al. [25]. They observed that the silver NPs effectively inhibited the growth of the tested yeasts at the concentrations below their cytotoxic limit against the tested human fibroblasts determined at a concentration equal to $30 \mathrm{mg} / \mathrm{L}$ of AgNPs.

\section{Anticancer}

AgNPs are attracting and currently known for a wide range of biomedical applications. AgNPs have shown to have good potential as an antitumor agent through various mechanisms involving oxidative stress, DNA damage, cell cycle arrest, apoptosis, or necrosis [26]. Lin et al. investigated the inhibition of autophagy enhances the anticancer activity of silver nanoparticles. They concluded and revealed a novel biological activity of AgNPs in inducing cytoprotective autophagy, and inhibition of autophagy may be a useful strategy for improving the efficacy of AgNPs in anticancer therapy [27].

\section{Antiviral}

In the present scenario, the increase in infectious diseases caused by viruses such as influenza A/H1N1, influenza A/H5N1, SARS-Cov, Dengue virus, HIV, HBV, and new encephalitis viruses is of prime concern. These infections can create havoc in no time because the rapid proliferation including glimpses of destruction caused by these viral infections have been observed in some of the countries and the most dangerous of these viral infections were bird flu, swine flu, and dengue which result in causing severe damage to health and wealth of human beings. AgNPs are famous for their antimicrobial activities; therefore researchers have diverted their attention and started evaluating the importance of AgNPs in controlling infectious diseases caused by pathogens and viruses. Nevertheless, the number of reported works using AgNPs for controlling viral infections is very low but still, it can pave the way for other researchers to show their interest in dealing with viral infections using AgNPs nanoparticle. In this regard, Elechiguerra et al. reported the effect of AgNPs on the HIV-1 virus. They found that the interaction between AgNPs and virus is size dependent (small-sized nanoparticles are more effective against these viruses) [28].

\section{Water Treatment}

AgNPs are long lasting, low cost with a controllable release rate of silver ions in comparison with bulk silver. Therefore, AgNPs are expected to play a crucial role in the food industry, water disinfection, and other applications related to disinfection. Drinking water purification is urgent needs of current era as normal tap water has a hazardous level of minerals, pesticides, microorganisms, etc.; in this regard, AgNPs could be cheap point-of-use material to purify drinking water. Several studies have been previously performed in water filtration applications to this context, generally 
to prevent bacterial fouling of membrane filters [29-31]. Additionally, Dankovich and Gray investigated bactericidal paper impregnated with silver nanoparticles for point-of-use water treatment [32]. In this study, silver-nanoparticle-containing papers were tested with respect to bacteria inactivation and silver leaching as suspensions of bacteria percolated through the paper. Therefore, AgNP sheets exhibited antibacterial properties toward suspensions of Escherichia coli and Enterococcus faecalis, with log reduction values in the effluent of over log 6 and $\log 3$, respectively. The silver loss from the AgNP sheets was minimal under current US EPA and WHO limit (0.1 ppm) for silver in drinking water. Thus, the percolation of bacterially contaminated water through paper embedded with silver nanoparticles could be an effective water treatment process in the near future.

\section{Protective Dressings}

At present protective dressings (bandages) functionalized with AgNPs are commercially available and are frequently used for medicinal purpose for curing various infections such as toxic epidermal necrolysis, Steven-Johnson syndrome, chronic ulcers, burn wounds, and pemphigus (blistering autoimmune disease). To develop protective dressings, typical bandages are coated on the surface of polyethylene or polythene layer. It has been recommended that these dressings have superior wound healing properties along with minimizing the healing time without any side effect as compared to the dressings without AgNPs treatment [33]. In a published study by Xing et al., it is observed that the dressing comprised of chitosan-AgNPs demonstrated superior healing rates contrary to the conventional wound dressing. Also, AgNPs in wound dressing can be helpful in eradicating problems related to skin infections [34].

\section{Catalysts}

With the development of nanoscience, nanocatalysis has clearly emerged in the end of the 1990s as a domain at the interface between homogeneous and heterogeneous catalysis, which offer unique solutions to answer the demanding conditions for improvement in catalysis processes. The main focus is to develop well-defined catalysts, which may include both metal nanoparticles and a nanomaterial as support. These nanocatalysts should be able to display the ensuing benefits of both homogeneous and heterogeneous catalysts, namely high efficiency and selectivity, stability and easy recovery/recycling [35]. Recently, it has been demonstrated experimentally that metallic nanoparticles have high catalytic characteristics for several chemical reactions such as hydrogenation, hydroformylation, and carbonylation. Nevertheless, in most cases, AgNPs would congregate during catalytic processes, because nanosized metal particles in the solution are active and prone to coalesce due to weak van der Waals forces and high surface energy unless they are protected. In this regard, surface modification using polymers, complex ligands, or surfactants is 
frequently used to stabilize metal catalysts [36]. Bastus et al. reported the highly monodisperse sodium citrate-coated spherical AgNPs with controlled sizes ranging from $10 \mathrm{~nm}$ to $200 \mathrm{~nm}$ have been synthesized by following a kinetically controlled seeded-growth approach via the reduction of silver nitrate by sodium citrate and tannic acid as chemical reducing agents. They found that as prepared AgNPs exhibited excellent reduction power with respect to the reduction of Rhodamine $B$ by sodium borohydride as a model reaction system [37]. Recent progress in synthetic chemistry AgNPs found a great interest because of their unique reactivity and selectivity, stability, as well as recyclability in catalytic reactions. In particular, a number of reactions can be catalyzed by AgNPs. Additionally, the strong potential of AgNPs in catalysis in the total synthesis of natural products and pharmaceutical molecules is highly under consideration currently. AgNPs catalyzed reactions widely utilized in various industrial applications including analgesic and antipyretic drugs, photographic develops, and corrosion inhibitors [38].

\section{Optical Properties}

Since AgNPs are extraordinarily efficient at absorbing and scattering light and, unlike many dyes and pigments, have a color that depends on the size and the shape of the particle. The strong interaction of nanosilver with light occurs because the conduction electrons on the metal surface undergo a collective oscillation when excited by light at specific wavelengths that are known as surface plasmon resonance (Fig. 3a, b) and results in unusually strong scattering and absorption properties. Therefore, there is growing popularity in the use of AgNPs as functional components in various optical products and sensors with optical properties. Choi and coworkers investigated the optical properties of the film prepared from chemically synthesized silver nanoparticles [39]. In this study, the extinction spectrum of the film shows three apparent peaks at $\sim 390 \mathrm{~nm}$ that come from isolated nanoparticles and the other two peaks at $\sim 360 \mathrm{~nm}$ and $\sim 580 \mathrm{~nm}$ that come from neighboring nanoparticles due
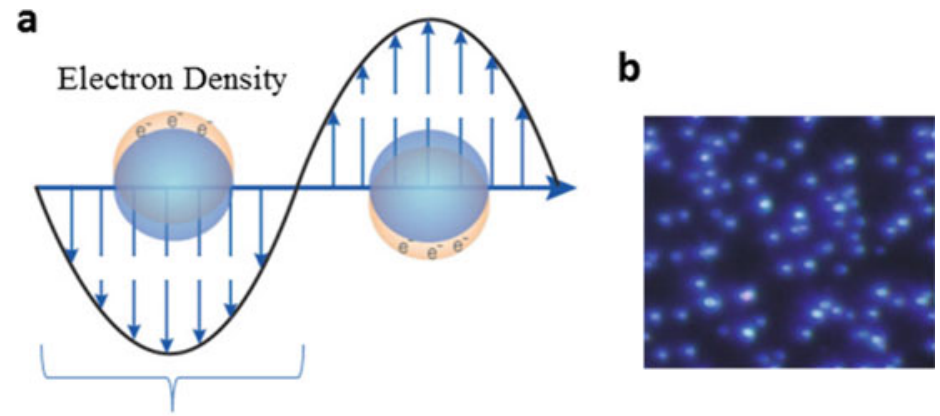

AgNP Sphere

Fig. 3 (a) Surface plasmon resonance (SPR) for AgNPs at specific wavelength. (b) Dark field microscopy image of AgNPs 
to interparticle dipole-dipole coupling (in-plane and out-of-plane contributions of linearly polarized components).

\section{Conclusion and Future Outlook}

In summary, different methods/processes are available to synthesize AgNPs. Of course, nanotechnology with emergence and use of metallic nanoparticles (MNPs) as novel bioactive functional agent that offers a unique way by means of currently used important active materials. The functional activities of MNPs owe to their high surface area to volume ratio and vast electronic, optical, magnetic, chemical, and physical properties and wide applications in various fields, such as in pharmaceutical and biomedical, biomedicine, catalysis, preparation of nanocomposites with tunable electrical conductivity, thermal conductivity, tensile strength, superior rigidity, hardness, and erosion resistance. These specific properties are used for manufacturing of satellite components, aircraft spares, industry parts, electronic microchips, etc to a significant extent. AgNPs with their unique chemical and physical properties are proving as an alternative for the development of new antimicrobial agents. They found diversified applications in the form of integrated materials including microbial resistant applications (antibacterial/antifungal/anticancer/antiviral), water treatment, protective dressings, catalysis, and optical devices. Presently, nanotechnology has opened new possibilities to the several sectors, especially in medicine, providing novel methods for the treatment of diseases which were previously difficult to target due to size restrictions. Therefore, these advantages make the present methods practically useful and potentially applicable to be commercialized at large scale, which are utilized in various fields, particularly in biomedical applications.

\section{References}

1. Gleiter H (2000) Nanostructured materials, basic concepts and microstructure. Acta Mater 48 (1): $1-12$

2. Dahl JA, Maddux BLS, Hutchison JE (2007) Toward greener nanosynthesis. Chem Rev 107:2228-2269

3. Braydich-Stolle L, Hussain S, Schlager J, Hofmann MC (2005) In vitro cytotoxicity of nanoparticles in mammalian germ line stem cells. Toxicol Sci 88:412-419

4. Hornyak GL, Dutta J, Tibbals HF, Rao A (2008) Introduction to nanoscience. CRC Press, Taylor and Francis LLC, New York

5. Konrad A, Herr U, Tidecks R, Samwer F (2001) Luminescence of bulk and nanocrystalline cubic yttria. J Appl Phys 90(7):3516-3523

6. Liang H, Li Z, Wang W, Wu Y, Xu H (2009) Highly surface-roughened "Flower-like" silver nanoparticles for extremely sensitive substrates of surface-enhanced Raman Scattering. Adv Mater 21(45):4614-4618

7. Rycenga M, Cobley CM, Zeng J, Li W, Moran CH, Zhang Q, Qin D, Xia Y (2011) Controlling the synthesis and assembly of silver nanostructures for plasmonic applications. Chem Rev 111(6):3669-3712 
8. Jia H, Zeng J, Song W, An J, Zhao B (2006) Preparation of silver nanoparticles by photoreduction for surface-enhanced Raman scattering. Thin Solid Films 2:281-287

9. Tsuji T, Okazaki Y, Tsuji M (2008) Photo-induced morphological conversions of silver nanoparticles prepared using laser ablation in water-enhanced morphological conversions using halogen etching. J Photochem Photobiol- A 194:247-253

10. Kshirsagar P, Sangaru SS, Malvindi MA, Martiradonna L, Cingolani R, Pomp PP (2011) Synthesis of highly stable silver nanoparticles by photoreduction and their size fractionation by phase transfer method. Colloids Surf A Physicochem Eng Asp 392:264-270

11. Zaarour M, El-Roz M, Dong B, Retoux R, Aad R, Cardin J, Dufour C, Gourbilleau F, Gilson JP, Mintova S (2014) Photochemical preparation of silver nanoparticles supported on zeolite crystals. Langmuir 30(21):6250-6256

12. Reetz MT, Helbig W (1994) Size-selective synthesis of nanostructured transition metal clusters. J Am Chem Soc 116(16):7401-7402

13. Rodriguez-Sanchez L, Blanco MC, Lopez-Quintela MA (2000) Electrochemical synthesis of silver nanoparticles. J Phys Chem B 104(41):9683-9688

14. Nasretdinova GR, Fazleeva RR, Osin YN, Gubaidullin AT, Yanilkin VV (2017) Methylviologen-mediated electrochemical synthesis of silver nanoparticles via the reduction of $\mathrm{AgCl}$ nanospheres stabilized by cetyltrimethylammonium chloride. Rus J Electrochemistry 1(53):25-38

15. Jokanović V, Spasić AM, Uskoković D (2004) Designing of nanostructured hollow $\mathrm{TiO}_{2}$ spheres obtained by ultrasonic spray pyrolysis. J Colloid Interface Sci 278(2):342-352

16. Pingali KC, Rockstraw DA, Deng S (2005) Silver nanoparticles from ultrasonic spray pyrolysis of aqueous silver nitrate. Aerosol Sci Technol 39(10):1010-1014

17. Bharde AA, Parikh RY, Baidakova M, Jouen S, Hannoyer B, Enoki T, Prasad BL, Shouche YS, Ogale S, Sastry M (2008) Bacteria-mediated precursor-dependent biosynthesis of superparamagnetic iron oxide and iron sulfide nanoparticles. Langmuir 24(11):5787-5794

18. Ahmed S, Ahmad M, Swami BL, Ikram S (2016) A review on plants extract mediated synthesis of silver nanoparticles for antimicrobial applications: a green expertise. J Adv Res 7(1):17-28

19. Kim JS, Kuk E, KN Y, Kim JH, Park SJ, Lee HJ, Kim SH, Park YK, Park YH, Hwang CY, Kim YK (2007) Antimicrobial effects of silver nanoparticles. Nanomedicine 3(1):95-101

20. He W, Liu X, Kienzle A, Müller WE, Feng Q (2016) In vitro uptake of silver nanoparticles and their toxicity in human mesenchymal stem cells derived from bone marrow. J Nanosci Nanotechnol 16(1):219-228

21. Sondi I, Salopek-Sondi B (2004) Silver nanoparticles as antimicrobial agent: a case study on E. coli as a model for Gram-negative bacteria. J Colloid Interface Sci 275(1):177-182

22. Morones JR, Elechiguerra JL, Camacho A, Holt K, Kouri JB, Ramírez JT, Yacaman MJ (2005) The bactericidal effect of silver nanoparticles. Nanotechnology 16(10):2346-2356

23. Sonker AS, Richa JP, Rajneesh VK (2017) Characterization and in vitro antitumor, antibacterial and antifungal activities of green synthesized silver nanoparticles using cell extract of Nostoc $s p$. strain HKAR-2. Can J Biotechnol 1(1):26-37

24. Kim KJ, Sung WS, Moon SK, Choi JS, Kim JG, Lee DG (2008) Antifungal effect of silver nanoparticles on dermatophytes. J Microb Biotechnol 18(8):1482-1484

25. Panáček A, Kolář M, Večeřová R, Prucek R, Soukupová J, Kryštof V, Hamal P, Zbořil R, Kvítek L (2009) Antifungal activity of silver nanoparticles against Candida spp. Biomaterials 30(31):6333-6340

26. Foldbjerg R, Dang DA, Autrup H (2011) Cytotoxicity and genotoxicity of silver nanoparticles in the human lung cancer cell line, A549. Arch Toxicol 85(7):743-750

27. Lin J, Huang Z, Wu H, Zhou W, Jin P, Wei P, Zhang Y, Zheng F, Zhang J, Xu J, Hu Y (2006) Inhibition of autophagy enhances the anticancer activity of silver nanoparticles. Autophagy 10 (11):2006-2020

28. Elechiguerra JL, Burt JL, Morones JR, Camacho-Bragado A, Gao X, Lara HH, Yacaman MJ (2005) Interaction of silver nanoparticles with HIV-1. J Nanobiotechnol 3(1):6

29. Yoon KY, Byeon JH, Park CW, Hwang J (2008) Antimicrobial effect of silver particles on bacterial contamination of activated carbon fibers. Environ Sci Technol 42(4):1251-1255 
30. Zodrow K, Brunet L, Mahendra S, Li D, Zhang A, Li Q, Alvarez PJ (2009) Polysulfone ultrafiltration membranes impregnated with silver nanoparticles show improved biofouling resistance and virus removal. Water Res 43(3):715-723

31. Li Q, Mahendra S, Lyon DY, Brunet L, Liga MV, Li D, Alvarez PJ (2008) Antimicrobial nanomaterials for water disinfection and microbial control: potential applications and implications. Water Res 42(18):4591-4602

32. Dankovich TA, Gray DG (2011) Bactericidal paper impregnated with silver nanoparticles for point-of-use water treatment. Environ Sci Technol 45(5):1992-1998

33. Ahamed M, AlSalhi MS, Siddiqui MK (2010) Silver nanoparticle applications and human health. Clin Chim Acta 411(23):1841-1848

34. Xing ZC, Chae WP, Baek JY, Choi MJ, Jung Y, Kang IK (2010) In vitro assessment of antibacterial activity and cytocompatibility of silver-containing PHBV nanofibrous scaffolds for tissue engineering. Biomacromolecules 11(5):1248-1253

35. Philippot K, Serp P (2012) Concepts in nanocatalysis. In: Serp P, Philippot K (eds) Nanomaterials in catalysis, 1 st edn. Wiley-VCH, Weinheim, pp 1-54

36. Jiang ZJ, Liu CY, Sun LW (2005) Catalytic properties of silver nanoparticles supported on silica spheres. J Phys Chem B 109(5):1730-1735

37. Bastús NG, Merkoçi F, Piella J, Puntes V (2014) Synthesis of highly monodisperse citratestabilized silver nanoparticles of up to $200 \mathrm{~nm}$ : kinetic control and catalytic properties. Chem Mater 26(9):2836-2846

38. Dong XY, Gao ZW, Yang KF, Zhang WQ, LW X (2015) Nanosilver as a new generation of silver catalysts in organic transformations for efficient synthesis of fine chemicals. Catal Sci Technol 5(5):2554-2574

39. Choi BH, Lee HH, Jin S, Chun S, Kim SH (2007) Characterization of the optical properties of silver nanoparticle films. Nanotechnology 18(7):075706 